\title{
Tumor Cell-Extracellular Matrix Interaction Modulates MMP-1 in Breast Cancer
}

\author{
Sekhar Pal1, Kirat Kumar Ganguly'1, Shyamsundar Mandal², Jaydip Biswas³, \\ Amitava Chatterjee ${ }^{1^{*}}$ \\ ${ }^{1}$ Department of Receptor Biology \& Tumor Metastasis, Chittaranjan National Cancer Institute, Kolkata, India \\ ${ }^{2}$ Department of Epidemiology \& Biostatistics, Chittaranjan National Cancer Institute, Kolkata, India \\ ${ }^{3}$ Department of Surgical Oncology, Chittaranjan National Cancer Institute, Kolkata, India \\ Email: * urenuschatterjee@gmail.com
}

Received 25 March 2015; accepted 24 April 2015; published 30 April 2015

Copyright (C) 2015 by authors and Scientific Research Publishing Inc.

This work is licensed under the Creative Commons Attribution International License (CC BY).

http://creativecommons.org/licenses/by/4.0/

c) (i) Open Access

\section{Abstract}

Increased MMP-1 expression in various tumor cells is significantly correlated with cancer progression. Enhanced secreted and intracellular level of MMP-1 is found in breast cancer cell line MDAMB-231 in presence of ECM glycoprotein fibronectin. To extrapolate this study into in vivo system, we observed the expression of MMP-1, fibronectin and $\alpha_{5} \beta_{1}$ integrin, which is the receptor for fibronectin, in breast cancer tissue samples. Expression of active form of MMP-1 was increased in tumor samples compared with the non-tumor counterpart. In some samples pro-MMP-1 was decreased but active form was increased in tumor part. The difference was more prominent in advanced stage tumor. ELISA showed appreciable increase in expression of $\alpha_{5}$ and $\beta_{1}$ integrins in tumor tissue in comparison to the non tumor counterpart in case of advanced stage tumor. Though there is no appreciable difference in fibronectin concentration, enhanced $\alpha_{5}, \beta_{1}$ integrin expression may mediate enhanced cell-ECM interaction to upregulate MMP-1 in tumor samples compared with the matched control.

\section{Keywords}

$\alpha_{5} \beta_{1}$ Integrin, Signaling, ECM, Fibronectin, MMP-1

\section{Introduction}

Breast cancer is the most commonly occurring cancer in women, comprising almost one third of all malignan-

"Corresponding author.

How to cite this paper: Pal, S., Ganguly, K.K., Mandal, S., Biswas, J. and Chatterjee, A. (2015) Tumor Cell-Extracellular Matrix Interaction Modulates MMP-1 in Breast Cancer. Journal of Cancer Therapy, 6, 375-382. 
cies in females [1]. Although the incidence of breast cancer has increased globally over the last several decades [2], the greatest increase has been in Asian countries [3]. Patients are also about one decade younger in developing countries than their counterparts in the developed nations. In India, breast cancer is the commonest cancer among women in urban registries of Delhi, Mumbai, Ahmedabad, Kolkata, and Trivandrum where it constitutes $>30 \%$ of all cancers in females [3]. The proportions of young patients ( $<35$ years old) vary from about $10 \%$ in developed to up to $25 \%$ in developing Asian countries, which contribute to poorer prognosis [4]. There is a significant difference in the survival rates in developed and developing countries mainly because of a lack of screening and early detection programmes, insufficient information regarding possible cancer markers in that ethnicity and inadequate resources for treatment [3]. Studies on tissue samples found several MMPs to be associated with breast cancer development and tumor progression [5]. In our laboratory, we have studied MMP-2 [6] and MMP-9 [7] in relation to breast cancer. In the present communication we have dealt with MMP-1.

MMP-1, being the most ubiquitously expressed interstitial collagenase, plays the prominent role in degradation of collagen, which is the body's most abundant protein [8], and assists metastasis. Like other MMPs, it consists of a pro domain, catalytic domain, a linker region and a hemopexin-like domain. Residues from both the catalytic domain and the hemopexin-like domain make interactions with the pro domain residues. The cleavage of the pro domain from the holoenzyme $(\sim 52 \mathrm{kDa})$ to form active enzyme ( $\sim 3 \mathrm{kDa})$ requires the hemopexin-like domain to undergoe a major displacement towards the catalytic domain, thus widening the cleft between the proteinase domain and the hemopexin-like domain on the active site face of the enzyme. This altered configuration makes the active site residues more accessible for native collagen [9]. Increased MMP-1 expression in various tumor cells is significantly correlated with the depth of tumor invasion, angiogenesis, lymphangiogenesis, poor prognosis and the presence of metastases [10]-[12]. Here we initially found enhanced secreted and intracellular level of MMP-1 in breast cancer cell line MDA-MB-231 in presence of extracellular matrix protein fibronectin (FN). To extrapolate our study into in vivo system, we observed the expression of MMP-1, FN and $\alpha_{5} \beta_{1}$ integrin receptor in breast cancer tissue samples.

\section{Patient}

Our present study involves 28 female breast cancer patients. They were referred to Chittaranjan National Cancer Institute, India, because of clinical breast lump, suspicious mammographic finding or a breast symptom (e.g. pain, nipple discharge) between 2008 and 2010. Women willing to participate in the project were interviewed and examined by a trained study nurse before any diagnostic procedure. The participation rate of patients with diagnosed breast cancer was $98 \%$. Thus the patient series should represent unselected typical breast cancer cases. After surgical treatment, the patients were offered adjuvant chemotherapy and/or hormonal therapy or radiotherapy, depending on the mode of the surgery, the patients' menopausal status, and the stage of the disease, according to the national guidelines. Stage was assessed by using the UICC classification. Patients with noninvasive carcinomas, a previous history of breast cancer, metastatic disease (stage-IV), or insufficient tumor material was excluded from the present study. Thus 28 patients with sufficient primary tumors and complete clinical histories were available for the present study. The mean age of the patients was 59.2 years old (median 56.8 years old; range, 23.3 - 91.6 years old). The mean follow up time was 55.0 months old (median 57.5 months old; range, 1.2 - 115.1 months old).

\section{Materials}

MDA-MB-231 cell line was purchased from National Centre for Cell Sciences (NCCS), Pune. Leibovitz's L-15 medium, Trypsin, Gentamycin, Fetal Bovine Serum (FBS) were purchased from GIBCO ${ }^{\mathrm{TM}}$-Invitrogen. Fibronectin (440 kDa), Protease Inhibitor Cocktail Tablets (complete, mini, EDTA-free) were purchased from Roche, Germany. Anti- $\alpha_{5}$, anti-MMP-1, anti- $\beta$-tubulin primary and all secondary antibodies were purchased from Santa Cruz Biotechnology, Inc. Anti- $\beta_{1}$ and anti-FN primary antibodies were purchased from Roche, Germany. Chemiluminescent substrate SuperSignal West Femto was purchased from Pierce, Thermo Fisher Scientific Inc. Avidin-biotinylated peroxidase complex reagent (vectastain ABC kit) was purchased from vector laboratories, Burlingame, CA. DAB substrate and stable peroxidase substrate buffer were purchased from Pierce Biotechnology, USA. Immobilon-P Membrane (PVDF) was purchased from Millipore, USA. TMB/ $\mathrm{H}_{2} \mathrm{O}_{2}$ was purchased from Bangalore Genei. 


\section{Methods}

\subsection{Collection of Tissue Samples}

Tissue specimens from tumor and respective adjacent non-tumor breast tissues of the same patient were collected from the operation theater during surgery. Tissues were stored at $-80^{\circ} \mathrm{C}$ and used for the further experiments.

\subsection{Protein Extraction from Tissue}

Tissues of tumor samples $(\mathrm{T})$ and respective adjacent non-tumor breast tissues $(\mathrm{N})$ were collected, extracted with NP-40 lysis buffer (50 mM Tris, $150 \mathrm{mM} \mathrm{NaCl}, 1 \%$ NP40, pH 8, Protease inhibitor cocktail tablets, $1 \mathrm{mM}$ sodium Orthovanadate and $1 \mathrm{mM}$ Sodium fluoride) and the protein content of the extracts were estimated by Lowry's method.

\subsection{Cell Line Maintenance and Treatment}

MDA-MB-231 (human breast cancer cell line) was obtained from National Centre for Cell Sciences (NCCS), Pune. This cell line was grown and maintained in Leibovitz's L-15 medium, containing $10 \% \mathrm{FBS}$ in a $5 \% \mathrm{CO}_{2}$ incubator at $37^{\circ} \mathrm{C}$. For the required treatment, $35 \mathrm{~mm}$ petridishes were coated with FN (20 $\left.\mu \mathrm{g} / \mathrm{ml}\right)$ in L15 SFCM (Serum Free Culture Media) 2 hrs prior to addition of cells. 300,000 cells/ml were added and incubated for overnight at $37^{\circ} \mathrm{C}, 5 \% \mathrm{CO}_{2}$.

\subsection{Western Blot}

Equal amount of protein ( $100 \mu \mathrm{g}$ each for $\mathrm{N}$ and $\mathrm{T}$ set) from tissue extracts were subjected to western blot analysis using anti-MMP-1 primary antibody (1 $\mu \mathrm{g} / 2 \mathrm{ml}$ dilution) and respective HRP-coupled secondary antibody (1 $\mu \mathrm{g} / 200 \mathrm{ml}$ dilution). Blots were developed by ECL method using SuperSignal West Femto according to manufacturer's protocol. Blots were reprobed with anti $\beta$-tubulin antibody as internal loading control (Ganguly et al. 2012). Comparative densitometric analysis for statistical calculations was done by Image J software (version $1.42 \mathrm{q})$.

\subsection{Statistical Analysis}

Statistical Analysis was performed with help of Epi Info (TM) 3.5.3. EPI INFO is a trademark of the Centers for Disease Control and Prevention (CDC).

Descriptive statistical analysis was performed to calculate the means with corresponding standard deviations (s.d.). One Way Analysis of variance (ANOVA) followed by Tukey's Test was performed with the help of Critical Difference (CD) or Least Significant Difference (LSD) at 5\% and 1\% level of significance to compare the mean values. $p \leq 0.05$ was taken to be statistically significant.

\subsection{Enzyme-Linked Immunosorbent Assay (ELISA)}

Equal amount of protein ( $5 \mu \mathrm{g}$ for $\alpha_{5}$ and $25 \mu \mathrm{g}$ for $\beta_{1}$ of each set of $\mathrm{N}$ and $\mathrm{T}$ ) was taken and coated in a 96 well flat bottom, sterile culture plate and subjected to ELISA against the blank of same volume of extraction buffer. In case of cell line experiment, culture supernatants of FN treated or untreated cells were used as sample and fresh SFCM was used as blank. After washing and blocking with blocking buffer (1\% BSA in PBS or phosphate buffer saline), wells were incubated ( $1 \mathrm{hr}$ at $37^{\circ} \mathrm{C}$ in humid chamber) with primary antibodies against $\alpha_{5}, \beta_{1}$ integrins and FN (anti-MMP-1 for the cell line culture supernatant experiment) in 1:1000 dilution (in dilution buffer of $0.1 \%$ BSA in PBS). After washing thrice with washing buffer (0.5\% NP-40, 0.5\% BSA in PBS), the wells were incubated with $\left(1 \mathrm{hr}\right.$ at $37^{\circ} \mathrm{C}$ in humid chamber) respective HRP coupled secondary antibody solution (in 1:2000 dilution in dilution buffer). Then wells were washed 6 times with washing buffer and substrate (TMB/ $\mathrm{H}_{2} \mathrm{O}_{2}$ ) was added. As colour appears, reaction was stopped by $1(\mathrm{M}) \mathrm{H}_{2} \mathrm{SO}_{4}$ and absorbance was measured at 450 $\mathrm{nm}$ in ELISA reader.

\subsection{Histology}

Both normal and tumor samples were fixed in $10 \%$ neutral buffered formalin, processed routinely and embedded 
in paraffin. The histologic diagnosis was confirmed by reviewing one to four original sections of the primary tumor. All tumors were simultaneously re-evaluated for histologic type and grade by one senior pathologist, who was unaware of the clinical data. The most representative blocks were selected for cutting into new $5 \mu \mathrm{m}$-thick sections for immunohistochemical analyses.

\subsection{Immunohistochemistry}

The immunostaining of MMP-1 was done using sequenza Immunostaining center (Shandon Scientific Limited, Astmoor, UK). In brief, the sections were de-parafinized in xylene, re-hydrated with graded EtOH, and distilled water. For better antigen retrieval, the samples were boiled three times for 15 minutes in boiling water in citrate buffer (10 mM, pH 6). Endogenous peroxidases were blocked by $0.3 \%$ hydrogen peroxide treatment for $30 \mathrm{mi}-$ nutes. The samples were washed with PBS (pH 7.2) and incubated in 2\% BSA for $1 \mathrm{hr}$ to prevent nonspecific antigen binding followed by incubation with the primary antibody for MMP-1 at a working dilution of 1:500 (in $1 \%$ BSA) for overnight at $4^{\circ} \mathrm{C}$. The samples were washed with PBS and secondary antibody (1:500 in 1\% BSA for one and half hr at room temperature) was applied. The slides were then incubated with the biotinylated secondary antibody followed by a wash and 30 minutes incubation in an avidin-biotinylated peroxidase complex reagent (ABC kit). Expressions were visualized with diaminobenzidin tetrahydrochloride (DAB) treatment. The slides were counterstained with Mayer's hematoxylin, dehydrated and mounted with DePex. A routinely processed breast cancer section without the primary antibody served as negative control at each staining series.

\section{Results}

\subsection{Enhanced Expression of MMP-1 in Fibronectin Treated Breast Cancer Cells}

MDA-MB-231 cells grown on FN coated $(20 \mu \mathrm{g} / \mathrm{ml})$ surface for overnight showed appreciable increase in MMP-1 expression in the culture supernatant and cell extract (Figure 1).

\subsection{Expression of Activated MMP-1 in Tumor Tissue}

Western blot analysis showed that the expression of active form of MMP-1 was increased in tumor samples compared to the non-tumor control. In many samples pro-MMP-1 ( 52 kDa) was also increased, in some samples pro-MMP-1 was decreased but active form ( 43 kDa) was increased in tumor tissue. Maximum samples of initial stage of the disease showed almost similar expression of MMP-1 in normal and tumor part. However, the same from advanced stage of cancer showed remarkably higher expression in tumor tissue (Table 1). Figure 2(a) shows the representative blots of MMP-1 expression. ANOVA showed that there were significant differences in MMP-1 expression level $\left(\mathrm{F}_{3,52}=5.24 ; p<0.01\right)$. As per the CD the mean of expression level of active MMP-1 in advance stage was significantly higher than that of initial stage $(p<0.01)$. But the mean of expression level of pro MMP-1 in advance stage was not significantly higher than that of initial stage $(p=0.08)$ (Figure 2(b)).

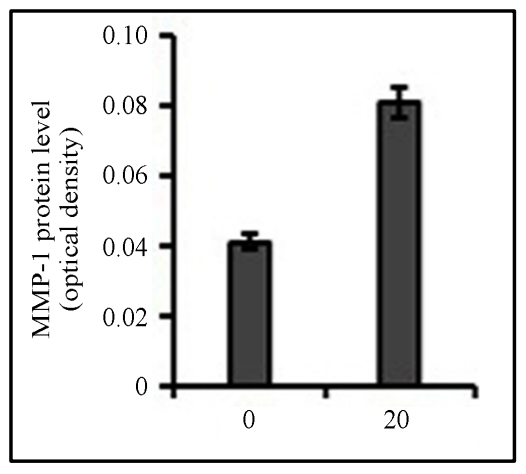

(a)

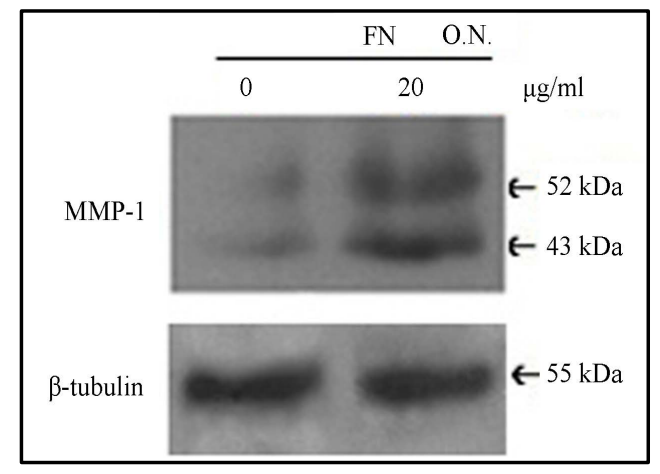

(b)

Figure 1. Effect of fibronectin (FN) on MMP-1 in MDA-MB-231 cells: MDA-MB-231 cells were grown in SFCM in absence (0) and in presence of $20 \mu \mathrm{g} / \mathrm{ml}$ coated FN for overnight (O.N.). The culture supernatants were subjected to ELISA (a) and cell extracts were subjected to western blot (b) for MMP-1. 
Table 1. Protein expression level (Mean \pm s.d.).

\begin{tabular}{cc}
\hline Stage & Mean \pm s.d. \\
\hline Active MMP-1 expression level in Initial Stage $(\mathrm{n}=13)$ & $0.11 \pm 0.02$ \\
Active MMP-1 expression level in Advance Stage $(\mathrm{n}=15)$ & $0.34 \pm 0.04$ \\
Pro MMP-1 expression level in Initial Stage $(\mathrm{n}=13)$ & $0.12 \pm 0.06$ \\
Pro MMP-1 expression level in Advance Stage $(\mathrm{n}=15)$ & $0.29 \pm 0.07$
\end{tabular}

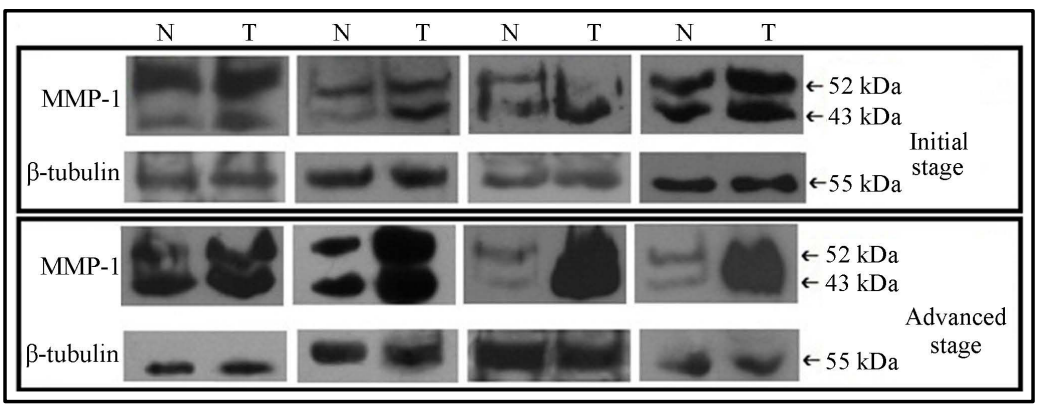

(a)

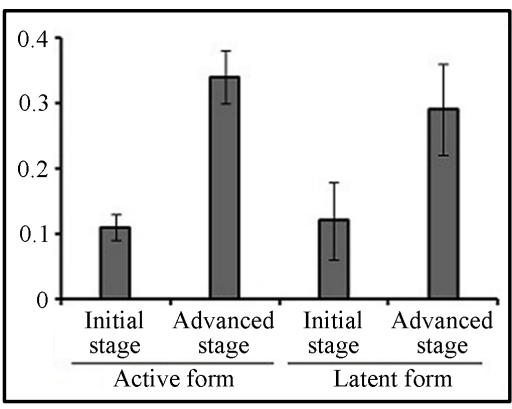

(b)

Figure 2. (a) Representative blot of MMP-1 in breast cancer specimen: Tissue homogenates from matched pairs of tumor (T) and non-tumor (N) specimens from the same patient were subjected to western blot analysis with anti-MMP-1 antibody and respective HRP-coupled secondary antibody for ECL method. Specimen of stage IIA and IIB patients are termed as initial stage and that of stage IIIA, IIIB, IIIC are termed as advanced stage. (b) Graphical representation of statistical analysis: Descriptive statistical analysis was performed to calculate the means with corresponding standard deviations (s.d.) of the data in Table 1.

\subsection{Upregulation of MMP-1 in Tumor Regions}

In tissue sections MMP-1 was found to be increased specifically in transformed cell clusters compared to the surrounding normal tissue regions, as visualized by immunohistochemistry and the view of a representative slide is given here (Figure 3).

\subsection{Enhanced Expression of $\alpha 5 \beta 1$ Integrins in Tumor Tissue}

ELISA showed appreciable increase in expression of $\alpha_{5}$ (Figure 4(a)), and $\beta_{1}$ (Figure 4(b)) integrins in tumor tissue in comparison to the non tumor counterpart in case of advanced stage tumor. This increase is not much prominent in initial stage tumor. Expression of fibronectin (FN), the ECM ligand of $\alpha_{5} \beta_{1}$ integrin receptor, does not seem to vary depending on the tumor stage between tumor and non tumor regions (Figure 4(c)).

\section{Discussion}

Interstitial collagens are essential structural components of all connective tissues and play an important role in cellular processes such as cell migration, proliferation and differentiation. MMP-1 is one of the key collagenases that are capable of cleaving interstitial fibrillar collagen. Although physiological collagenolysis is required for several biological processes such as embryogenesis, tissue remodeling, angiogenesis, wound healing, etc., uncontrolled degradation of collagen may result in pathological conditions like cancer, arthritis, fibrosis [9]. MMP-1 has been claimed as a candidate marker that may be useful for identification of breast lesions that can develop into cancer [13]. In our study on tissue homogenates of breast cancer patients, MMP-1 expression was found to be enhanced appreciably in tumor region than the adjacent non-tumor breast tissues. Active form of MMP-1 was more prominent in tumor tissue especially in advanced staged tumor. Our immunohistochemistry data further strengthen the fact of increased expression of MMP-1 in tumor section than the surrounding adjacent non-tumor regions.

Interaction of tumor cells with extracellular matrix (ECM) proteins, e.g. fibronectin, laminin, etc., increases tumorigenicity by enhancing expression and/or activation of MMPs, cell migration, invasion, metastasis, etc. 


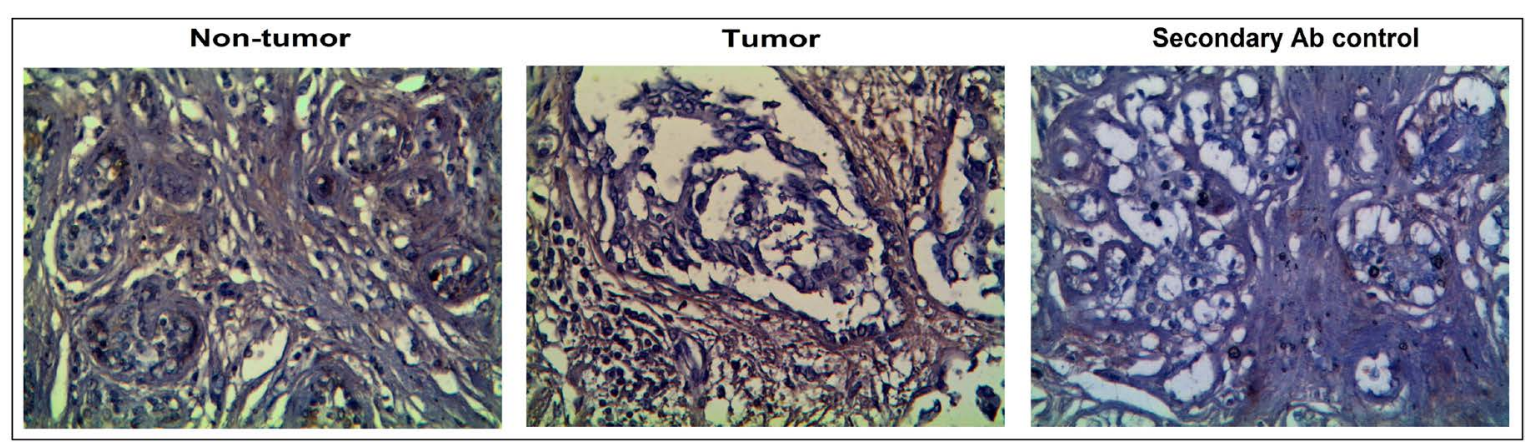

Figure 3. Immunohistochemistry with anti-MMP-1 antibody in breast cancer tissue sections: Tissue sections from breast tissue of the tumor and non-tumor region of the same patient was subjected to immunohistochemical analysis for MMP-1. Expression of MMP-1 is detected by brown stain of DAB. Secondary Ab control (without anti-MMP-1 primary $\mathrm{Ab}$ ) was done to exclude the possibility of non-specific attachment of secondary antibody.

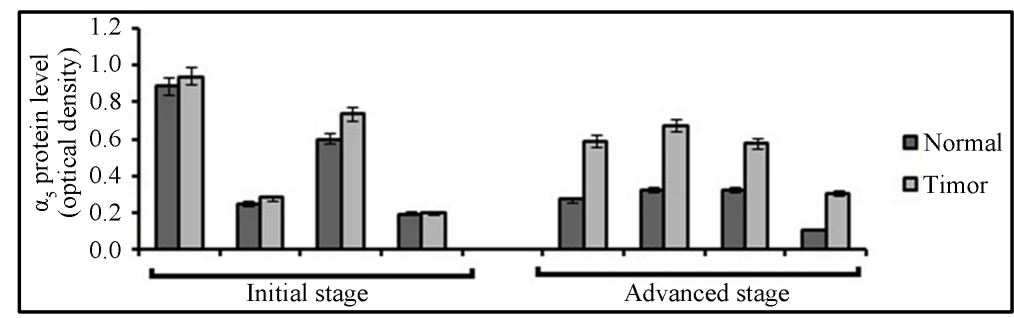

(a)

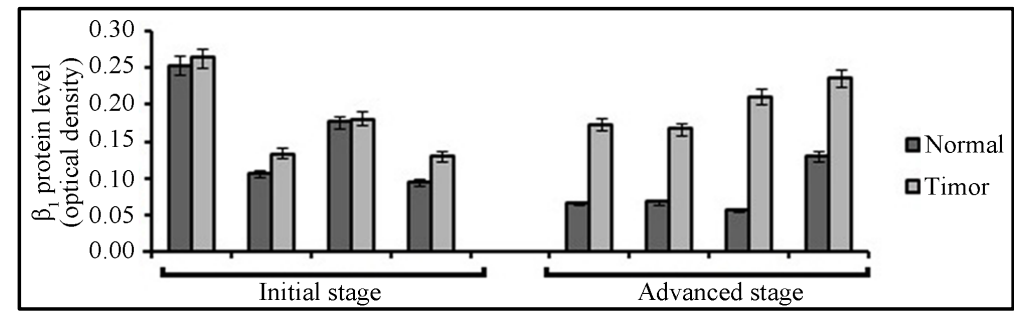

(b)

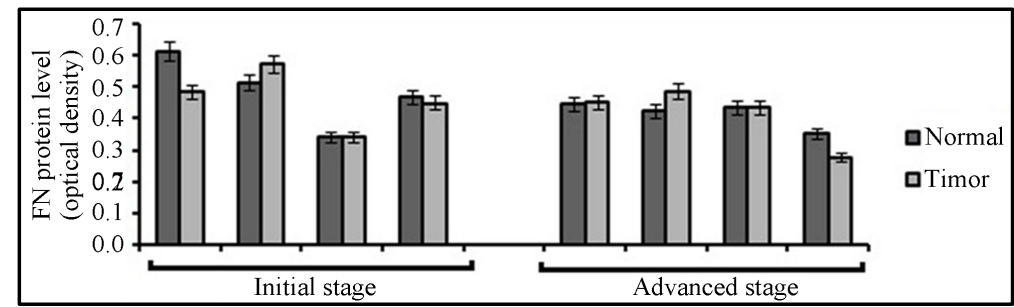

(c)

Figure 4. Representative ELISA of $\alpha_{5}$ integrins, $\beta_{1}$ integrins and FN in breast cancer specimen: Tissue homogenates from matched pairs of tumor and nontumor specimens from the same patient were subjected to ELISA with anti- $\alpha_{5}$ integrin (a), anti- $\beta_{1}$ integrin (b) anti-fibronectin (c) antibodies.

[14]-[19]. Among the several cell surface integrin receptors, $\alpha_{5} \beta_{1}$ is a major FN receptor on most cells and play important roles in mediating downstream signaling to regulate various effecter functions [20]. This integrin mediated signaling was found to play a very crucial role in MMP-1 production stimulated by fibronectin-specific sequence [21]. In our study of breast cancer samples, tissue homogenates from tumor regions showed elevated level of MMP-1, $\alpha_{5}$ and $\beta_{1}$ integrins in comparison to the adjacent non tumor regions. These effects were more prominent in advanced stage tumor than the initial stage tumor samples. However, FN level was independent of tumor stage between tumor and non-tumor regions. Upregulation of $\alpha_{5} \beta_{1}$ integrin may be responsible for en- 
hanced MMP-1 activation by transducing signal initiated by ligand-receptor interaction.

Therefore it may be concluded that in breast cancer sample, expression and activation of MMP-1 is appreciably enhanced in comparison to the matched control. In our model experiment with breast cancer cell line MDAMB-231, similar effect was observed. Enhanced $\alpha_{5}, \beta_{1}$ integrin expression may mediate enhanced cell-ECM interaction to upregulate MMP-1 in tumor samples compared with the matched control. The difference is more appreciable in advanced stage tumor than initial stage. The study of integrin mediated regulation of MMP-1 may be exploited in clinical and therapeutic management of cancer.

\section{Acknowledgements}

The authors wish to express their thanks to Director, Chittaranjan National Cancer Institute, for continuous inspiration and financial support.

\section{References}

[1] Richie, R.C. and Swanson, J.O. (2003) Breast Cancer: A Review of the Literature. Journal of Insurance Medicine, 35, 85-101.

[2] Anderson, B.O. and Jakesz, R. (2008) Breast Cancer Issues in Developing Countries: An Overview of the Breast Health Global Initiative. World Journal of Surgery, 32, 2578-2585. http://dx.doi.org/10.1007/s00268-007-9454-z

[3] Khokhar, A. (2012) Breast Cancer in India: Where Do We Stand and Where Do We go? Asian Pacific Journal of Cancer Prevention, 13, 4861-4866. http://dx.doi.org/10.7314/APJCP.2012.13.10.4861

[4] Agarwal, G., Pradeep, P.V., Aggarwal, V., Yip, C.H. and Cheung, P.S. (2007) Spectrum of Breast Cancer in Asian Women. World Journal of Surgery, 31, 1031-1040. http://dx.doi.org/10.1007/s00268-005-0585-9

[5] Kohrmann, A., Kammerer, U., Kapp, M., Dietl, J. and Anacker, J. (2009) Expression of Matrix Metalloproteinases (MMPs) in Primary Human Breast Cancer and Breast Cancer Cell Lines: New Findings and Review of The Literature. BMC Cancer, 9, 188. http://dx.doi.org/10.1186/1471-2407-9-188

[6] Sil, H., Moulik, S., Singha, S., Mandal, S.S., Biswas, J. and Chatterjee, A. (2015) Activation of Matrix Metalloproteinase 2(MMP-2) in Breast Cancer Progression. Journal of Tumor, 3, 292-304.

[7] Nanda, D.P., Sil, H., Moulik, S., Biswas, J, Mandal, S.S. and Chatterjee, A. (2013) Matrix Metalloproteinase-9 as a Potential Tumor Marker in Breast Cancer. Journal of Environmental Pathology, Toxicology and Oncology, 32, 115129.

[8] Rutter, J.L., Mitchell, T.I., Buttice, G., Meyers J, Gusella JF, Ozelius, L.J. and Brinckerhoff, C.E. (1998) A Single Nucleotide Polymorphism in the Matrix Metalloproteinase-1 Promoter Creates an Ets Binding Site and Augments Transcription. Cancer Research, 58, 5321-5325.

[9] Iyer, S., Visse, R., Nagase, H. and Acharya, K.R. (2006) Crystal Structure of an Active Form of Human MMP-1. Journal of Molecular Biology, 362, 78-88. http://dx.doi.org/10.1016/j.jmb.2006.06.079

[10] Murray, G.I., Duncan, M.E., O’Neil, P., McKay, J.A., Melvin, W.T. and Fothergill J.E. (1998) Matrix Metalloproteinase-1 Is Associated with Poor Prognosis in Oesophageal Cancer. The Journal of Pathology, 185, 256-261. http://dx.doi.org/10.1002/(SICI)1096-9896(199807)185:3<256::AID-PATH115>3.0.CO;2-A

[11] Murray, G.I., Duncan, M.E., O’Neil, P., Melvin, W.T. and Fothergill, J.E. (1996) Matrix Metalloproteinase-1 Is Associated with Poor Prognosis in Colorectal Cancer. Nature Medicine, 2, 461-462. http://dx.doi.org/10.1038/nm0496-461

[12] Shiozawa, J., Ito, M., Nakayama, T., Nakashima, M., Kohno, S. and Sekine, I. (2000) Expression of Matrix Metalloproteinase-1 in Human Colorectal Carcinoma. Modern Pathology, 13, 925-933. http://dx.doi.org/10.1038/modpathol.3880169

[13] Poola, I., DeWitty, R.L., Marshalleck, J.J., Bhatnagar, R., Abraham, J. and Leffall, L.D. (2005) Identification of MMP1 as a Putative Breast Cancer Predictive Marker by Global Gene Expression Analysis. Nature Medicine, 11, 481-483. http://dx.doi.org/10.1038/nm1243

[14] Das, S., Banerji, A., Frei, E. and Chatterjee, A. (2008) Rapid Expression and Activation of MMP-2 and MMP-9 upon Exposure of Human Breast Cancer Cells (MCF-7) to Fibronectin in Serum Free Medium. Life Sciences, 82, 467-476. http://dx.doi.org/10.1016/j.lfs.2007.12.013

[15] Maity, G., Choudhury, P.R., Sen, T., Ganguly, K.K., Sil, H. and Chatterjee, A. (2011) Culture of Human Breast Cancer Cell Line (MDA-MB-231) on Fibronectin-Coated Surface Induces Pro-Matrix Metalloproteinase-9 Expression and Activity. Tumor Biology, 32, 129-138. http://dx.doi.org/10.1007/s13277-010-0106-9

[16] Maity, G., Sen, T. and Chatterjee, A. (2011) Laminin Induces Matrix Metalloproteinase-9 Expression and Activation in Human Cervical Cancer Cell Line (SiHa). Journal of Cancer Research and Clinical Oncology, 137, 347-357. 
http://dx.doi.org/10.1007/s00432-010-0892-x

[17] Pal, S., Ganguly, K.K. and Chatterjee, A. (2013) Extracellular Matrix Protein Fibronectin Induces Matrix Metalloproteinases in Human Prostate Adenocarcinoma Cells PC-3. Cell Communication and Adhesion, 20, 105-114. http://dx.doi.org/10.3109/15419061.2013.833193

[18] Pal, S., Moulik, S., Dutta, A. and Chatterjee, A. (2014) Extracellular Matrix Protein Laminin Induces Matrix Metalloproteinase-9 in Human Breast Cancer Cell Line MCF-7. Cancer Microenvironment, 7, 71-78. http://dx.doi.org/10.1007/s12307-014-0146-6

[19] Sen, T., Dutta, A., Maity, G. and Chatterjee, A. (2010) Fibronectin Induces Matrix Metalloproteinase-9 (MMP-9) in Human Laryngeal Carcinoma Cells by Involving Multiple Signaling Pathways. Biochimie, 92, 1422-1434. http://dx.doi.org/10.1016/j.biochi.2010.07.005

[20] Pal, S., Ganguly, K.K., Moulik, S. and Chatterjee, A. (2012) Modulation of MMPs by Cell Surface Integrin Receptor $\alpha_{5} \beta_{1}$. Anti-Cancer Agents in Medicinal Chemistry, 12, 726-732. http://dx.doi.org/10.2174/187152012802650183

[21] Jia, Y., Zeng, Z.Z., Markwart, S.M., Rockwood, K.F., Ignatoski, K.M., Ethier, S.P. and Livant, D.L. (2004) Integrin Fibronectin Receptors in Matrix Metalloproteinase-1-Dependent Invasion by Breast Cancer and Mammary Epithelial Cells. Cancer Research, 64, 8674-8681. http://dx.doi.org/10.1158/0008-5472.CAN-04-0069 